\title{
RELATIVISTIC CONTRIBUTIONS TO ENERGIES OF \\ HIGHLY IONIZED ATOMS
}

\author{
RALPH SNYDER
}

Dept. of Applied Mathematics and Theoretical Physics, The Queen's University of Belfast, Belfast, N. Ireland

\section{Introduction}

Historically, the first observation of a relativistic effect in atomic spectra was probably the discovery by Fraunhofer of the splitting of the Na I doublet in the solar spectrum. Thus theoretical understanding of these effects has long been important for interpreting astrophysical spectra, and it is especially important for the ultraviolet and $\mathrm{X}$-ray spectra which form our subject today, for these often arise in highly ionized atoms. Because the relative importance of relativistic to non-relativistic terms is proportional to $Z^{2}$, where $Z$ is the nuclear charge, relativistic effects often play a major role in such atoms.

\section{Theory}

I would like to describe a theoretical method which I have developed for approximating the relativistic portion of the energy in many-electron atoms or ions. First it will be helpful to recall very briefly the known result in the much simpler oneelectron case. There the Dirac equation can be solved exactly and shows that the relativistic energy through order $\alpha^{2}$, where $\alpha$ is the fine structure constant, is given by

$$
E^{\mathrm{Rel}}=\alpha^{2} \varepsilon_{n j} Z^{4}, \quad \varepsilon_{n j}=-\frac{1}{2 n^{4}}\left(\frac{n}{j+\frac{1}{2}}-\frac{3}{4}\right) \mathrm{AU} .
$$

In a well-known textbook exercise, one can show that this result can be decomposed into a spin-orbit term plus a mass-velocity term plus the Darwin term.

In the many-electron case, to which we now turn, just this sort of decomposition forms the basis for conventional calculations of relativistic effects, which most commonly consist of evaluating spin-orbit parameters with variational wave functions. Such an approach takes no account of the known one-electron result given by Equation (1) which, after all, underlies the many-electron case as well. In contrast, the method I want to describe incorporates Equation (1) as a starting point by writing the many-electron energy as a sum of screened one-electron terms,

$$
E^{\mathrm{Rel}}=\alpha^{2} \sum_{i} q_{i} \varepsilon_{i}\left(Z-\sigma_{i}\right)^{4}, \quad i=1 \frac{1}{2}, 2 s_{2}, 2 p \frac{1}{2}, 2 p \frac{3}{2}, \ldots
$$

where $q_{i}$ is the number of electrons of type $i$. Equation (2) gives the total relativistic energy for a $j j$-coupled state; this can easily be transformed to $L S$ coupling where desired.

The screenings, $\sigma_{i}$, now represent the interelectron effects and, if the method is to 
be a precise one, must be carefully specified. Many-electron effects can be broken up into sums of two-electron interactions and we may take this into account by deriving each $\sigma_{i}$ from a two-electron screening matrix $\sigma(i \mid j)$, according to the equation

$$
\sigma_{i}=\sum_{j}\left(q_{j}-\delta_{i j}\right) \sigma(i \mid j)
$$

Equation (3) may be said to describe the screening of an electron of type $i$ by each of the $q_{j}$ electrons of type $j$ by an amount $\sigma(i \mid j)$, with self-screening prohibited by the Kronecker delta $\delta_{i j}$. Now our scheme will be complete once we determine the elements of the screening matrix $\sigma(i \mid j)$, by studying the two-electron interactions.

To do this, we will make use of the relativistic $Z$-expansion theory (Layzer and Bahcall, 1962; Dalgarno and Stewart, 1960). If we expand Equation (2) in powers of $Z$, we have in fact a $Z$-expansion of the relativistic energy, and with the zero-order $\left(\sim \alpha^{2} Z^{4}\right)$ term given correctly as a sum of hydrogenic terms. It is natural to try to extend the validity beyond zero-order and so we will determine $\sigma(i \mid j)$ by demanding that, for all two-electron cases, Equation (2) also reproduce correctly the first-order and second-order $Z$-expansion coefficients. Matching terms, we see that this demand gives us two simple algebraic equations from which to determine $\sigma(a \mid b)$ and $\sigma(b \mid a)$,

$$
\begin{aligned}
& q_{a} \varepsilon_{a} \sigma(a \mid b)+q_{b} \varepsilon_{b} \sigma(b \mid a)=-\frac{1}{4} E_{1}^{a b} \\
& q_{a} \varepsilon_{a} \sigma^{2}(a \mid b)+q_{b} \varepsilon_{b} \sigma^{2}(b \mid a)=\frac{1}{6} E_{2}^{a b},
\end{aligned}
$$

where $a$ and $b$ label the two electrons. We need only consider the $(2 j+1)$-weighted average of the $j j$-configurations.

Values of $E_{1}^{a b}$ can be obtained from the calculations of Doyle (1969) for all cases involving electrons of principal quantum number $n=1$ and 2, so the right-hand side of Equation (4) is known. Good estimates of $E_{2}^{a b}$ are not yet available so, as a stopgap measure, we will satisfy (5) implicitly by imposing two conditions derived from the most naive picture of $\sigma(a \mid b)$ as a screening of electron $a$ by electron $b$,

$$
\begin{aligned}
& \sigma(a \mid b)=0 \text { if } n_{a}<n_{b}, \quad \text { and } \\
& \sigma(a \mid b)=\sigma(b \mid a) \text { if } n_{a}=n_{b} .
\end{aligned}
$$

Equation (6) is suggested by the fact that an electron's radial charge distribution is largely inferior to that of electrons with larger values of $n$; that it is generally similar to that of electrons with the same value of $n$ suggests Equation (7). Equations (4), (6) and (7) can now be solved, leading to the screening matrix shown in Table I.

TABLE I

\begin{tabular}{lllll}
\multicolumn{5}{c}{ Screening matrix $\sigma(i \mid j)$} \\
$i^{j}$ & $1 s^{\frac{1}{2}}$ & $2 s \frac{1}{2}$ & $2 p \frac{1}{2}$ & $2 p \frac{3}{2}$ \\
\hline $1 s^{\frac{1}{2}}$ & 0.48014 & 0 & 0 & 0 \\
$2 s^{\frac{1}{2}}$ & 0.64045 & 0.19484 & 0.20332 & 0.22340 \\
$2 p \frac{1}{2}$ & 0.98821 & 0.20332 & 0.29458 & 0.27732 \\
$2 p p^{\frac{3}{2}}$ & 1.45130 & 0.22340 & 0.27732 & 0.40896 \\
\hline
\end{tabular}


To review very briefly, we have constructed $\sigma(i \mid j)$ so that it will correctly reproduce the first three terms in the $Z$-expansion for all two-electron cases. Having done so, we propose that for any case with three or more electrons Equations (2) and (3) prescribe $E^{\mathrm{Re} 1}$ as a simple analytic function of $\sigma(i \mid j)$, the nuclear charge $Z$, the configuration quantum numbers $q_{i}$, and the one-electron coefficients $\varepsilon_{i}$. One indication that Equations (2) and (3) do this successfully is given by the important result, easy to prove, that our scheme now automatically reproduces the exact value of the firstorder term $\left(\sim \alpha^{2} Z^{3}\right)$ for any many-electron case.

For another indication of the results we may expect from this method we consider its predictions of the doublet splittings in $1 s^{2} 2 p^{2} P, 1 s^{2} 2 s^{2} 2 p^{2} P$, and in $1 s^{2} 2 s^{2} 2 p^{5} P$. In Table II we compare the $\sigma(i \mid j)$ results with experiment and with the spin-orbit calculations of Froese (1967) and of Condon and Odabasi (1966). The empirical values are taken from Edlén $(1964,1969)$ and, following the presentation there, all theoretical results have been reduced to $S(Z)$, defined by

$$
\Delta E^{\mathrm{Re} 1}=\Delta E_{0}^{\mathrm{Rel}}(Z-S)^{4} .
$$

Table II shows that the $\sigma(i \mid j)$ results are of similar accuracy to those of Froese in the LiI and FI sequences, are less accurate than Froese's in the BI sequence, and are much more accurate than those of Condon and Odabasi in all cases. I find these results encouraging, especially since they come from such an elementary version of $\sigma(i \mid j)$.

\section{Conclusion}

In conclusion, let me summarize some of the advantages which such an approach can offer. (1) It always provides an estimate of the total relativistic energy, rather than just, say, the spin-orbit part. (2) It treats an isoelectronic sequence as an entity, rather than dealing with each ion as a distinct problem. (3) It is an extremely simple method, substituting the elementary arithmetic of Equations (2) and (3) where the conventional approach solves coupled integro-differential equations, using the solutions to perform numerical integrations. This simplicity is of course computationally convenient and, more importantly, uncovers an unexpected simplicity in the structure of the relativistic energies themselves. Apparently a good understanding of the relativistic energies of all many-electron systems which involve electrons with $n=1$ or 2 requires the knowledge of only twenty numbers: the four $\varepsilon_{i}$ and the sixteen elements of $\sigma(i \mid j)$. Conventional methods do not recognize this simplicity and recover its implications (if at all) only after extensive numerical computation. (4) When one turns from doublets to triplets, quartets, etc., there arises the question of the relative intervals of the fine structure levels, a question which spin-orbit theory answers with the Landé interval rule. There are significant departures from the Landé rule even in the limit of $L S$-coupling and these can be accounted for by a $Z$-expansion method (Snyder, 1970), in particular, by the $\sigma(i \mid j)$ method slightly modified to allow for the splitting of the $j j$-configurations in these cases. (5) Finally, there is at least the hope that when 
methods superior to Equations (6) and (7) are available for estimating the $E_{2}^{a b}$, the resulting $\sigma(i \mid j)$ will lead to improved accuracy in theoretical predictions. Such an advance in our understanding of relativistic atomic energies is badly needed, for theory has long lagged far behind experiment in this field. At present for example, the most sophisticated theoretical calculation (Froese, 1967) of the splitting of Fraunhofer's $D$ lines, with which we began, is in error by $33 \%$.

TABLE II

$S(Z)$ for doublet splittings

$\begin{array}{lll}\text { Empirical } \sigma(i \mid j) & \text { Froese } & \begin{array}{l}\text { Condon } \\ \text { and } \\ \text { Odabasi }\end{array} \\ & \end{array}$

Lit Sequence: $1 s^{2} 2 p^{2} P$

$\begin{array}{lllll}3 & 2.020 & 1.918 & 2.100 & 1.738 \\ 4 & 1.937 & 1.870 & 2.003 & 1.594 \\ 5 & 1.982 & 1.841 & 1.942 & 1.523 \\ 6 & 1.856 & 1.822 & 1.904 & 1.481 \\ 7 & 1.844 & 1.810 & 1.878 & 1.454 \\ 8 & 1.822 & 1.801 & 1.858 & 1.434 \\ 9 & 1.811 & 1.794 & 1.844 & 1.420\end{array}$

BI Sequence: $1 s^{2} 2 s^{2} 2 p^{2} P$

$\begin{array}{rllll}5 & 2.450 & 2.171 & 2.461 & 2.037 \\ 6 & 2.357 & 2.159 & 2.358 & 1.929 \\ 7 & 2.308 & 2.149 & 2.303 & 1.873 \\ 8 & 2.278 & 2.142 & 2.268 & 1.838 \\ 9 & 1.257 & 2.136 & 2.243 & 1.813 \\ 10 & 2.241 & 2.131 & 2.224 & 1.795 \\ 11 & 2.229 & 2.127 & 2.209 & 1.780 \\ 12 & 2.220 & 2.124 & 2.198 & 1.769 \\ 13 & 2.213 & 2.122 & 2.187 & 1.760 \\ 14 & 2.206 & 2.119 & 2.180 & 1.752 \\ 15 & - & 2.117 & 2.173 & 1.746 \\ 16 & 2.197 & 2.116 & 2.167 & 1.740 \\ 17 & - & 2.114 & 2.162 & 1.735 \\ 18 & 2.189 & 2.113 & 2.158 & 1.731\end{array}$

FI Sequence: $1 s^{2} 2 s^{2} 2 p^{5} 2 P$

\begin{tabular}{rrrrr}
9 & 3.236 & 3.201 & 3.248 & 2.757 \\
10 & 3.208 & 3.185 & 3.220 & 2.731 \\
11 & 3.188 & 3.171 & 3.200 & 2.712 \\
12 & 3.172 & 3.160 & 3.185 & 2.698 \\
13 & 3.160 & 3.150 & 3.173 & 2.686 \\
14 & 3.150 & 3.142 & 3.162 & 2.677 \\
15 & 3.141 & 3.134 & 3.153 & 2.669 \\
16 & 3.134 & 3.129 & 3.145 & 2.662 \\
17 & 3.128 & 3.124 & 3.139 & 2.656 \\
18 & 3.123 & 3.120 & 3.134 & 2.651 \\
19 & 3.118 & 3.116 & 3.129 & 2.647 \\
20 & 3.114 & 3.112 & 3.124 & 2.642 \\
\hline
\end{tabular}




\section{Acknowledgements}

This research was supported by the Advanced Research Projects Agency of the United States Department of Defense and was monitored by the Office of Naval Research under Contract No. N00014-69-C-0035.

\section{References}

Condon, E. U. and Odabasi, H.: 1966, in Quantum Theory of Atoms, Molecules and the Solid State (ed. by P. O. Lowdin), Academic Press, New York, p. 185.

Dalgarno, A. and Stewart, A. L.: 1960, Proc. Phys. Soc. 75, 441.

Doyle, H. T.: 1969, in Advances in Atomic and Molecular Physics 5, (ed. by D. R. Bates and I. Estermann), Academic Press, New York, p. 337.

Edlén, B.: 1964, 'Atomic Spectra' in Handbuch der Physik 27, Springer-Verlag, Berlin, p. 80.

Edlén, B.: 1969, Solar Phys. 9, 439.

Froese, C.: 1967, Can. J. Phys. 45, 1501.

Layzer, D. and Bahcall, J.: 1962, Ann. Phys. 17, 177.

Snyder, R.: 1970, J. Phys. B (2) 3, L77. 